Check for updates

Cite this: Chem. Commun., 2019, 55, 12196

Received 23rd July 2019,

Accepted 5th September 2019

DOI: 10.1039/c9cc05693a

rsc.li/chemcomm

\section{Solvent-free functionalisation of graphene oxide with amide and amine groups at room temperature $\dagger$}

\author{
Stefania Sandoval, (D) Amparo Fuertes (D) and Gerard Tobias (D) *
}

\begin{abstract}
A new solvent free protocol is presented to introduce amide and amine functionalities ( $\mathrm{N}$-aliphatic groups) onto graphene oxide in an energy efficient manner. Nitrogen contents of 3.6 wt\% are obtained in only 5 minutes at room temperature by using ammonia gas as the nitrogen source for the ammonolysis of graphene oxide.
\end{abstract}

Functionalisation and doping are widely used strategies for modifying the properties of 2D carbon-based nanostructures. ${ }^{1}$ The formation of aliphatic moieties on the surface of graphene not only minimises processing issues like wrinkling, restacking, aggregation and low dispersibility in common solvents, but also allows tuning the optical, (bio)chemical and mechanical properties of the material. ${ }^{2}$ Moreover, functionalised GO derivatives can act as former materials for building more complex nanostructures. ${ }^{3}$ Graphene oxide (GO) has attracted interest due to its high hydrophilicity and water dispersibility that facilitate its processability. GO is composed of a variety of O-based moieties such as epoxy, hydroxyl or carboxylic groups that can be further derivatised. ${ }^{4}$ Both $\mathrm{N}$-doping and functionalisation with $\mathrm{N}$-aliphatic groups expand the range of properties and applications of graphene-based materials. ${ }^{5}$ Reduced graphene oxide (RGO) bearing N-aliphatic groups presents a higher dispersibility in aqueous solutions ${ }^{6}$ and organic solvents ${ }^{7}$ than GO. On the other hand, an enhanced thermal oxidation stability of RGO has been reported by nitrogen doping. ${ }^{8}$ The presence of $\mathrm{N}$-aliphatic moieties on the conjugated lattice is of interest for instance in the biomedical field, ${ }^{9}$ for water treatment, ${ }^{10}$ heavy-metal detection ${ }^{11}$ and catalysis. ${ }^{12} \mathrm{~A}$ variety of protocols have been developed to introduce amides and amines onto GO, often requiring pre-treatments ${ }^{13}$ or the presence of a catalyst to increase its reactivity. ${ }^{14} \mathrm{~N}$ loadings in the range of 1-7.9 wt\% have been reported when using organic solvents, ${ }^{7}$ high temperatures,${ }^{15}$ or multistep and complex synthetic strategies. ${ }^{7,12,16}$ In some cases, the resulting samples contain a mixture of aliphatic species ( $\mathrm{N}$-functionalisation) and $\mathrm{N}$ introduced into the lattice

Institut de Ciència de Materials de Barcelona (ICMAB-CSIC), Campus de la UAB, 08193 Bellaterra (Barcelona), Spain. E-mail: gerard.tobias@icmab.es

$\dagger$ Electronic supplementary information (ESI) available: Experimental details and additional characterisation of the prepared samples. See DOI: 10.1039/c9cc05693a
(N-doping). ${ }^{17}$ Herein, we present a solvent-free approach at room temperature that allows the introduction of amides and amines in a simple, fast and energy efficient manner via the treatment of GO with ammonia gas. Remarkably, the total amount of $\mathrm{N}$ introduced onto GO (3.6-5.2 wt\%) is in line with the values obtained with previously reported methods. The samples were prepared by direct reaction of GO (from a modified Hummers' method) ${ }^{6}$ with ammonia gas at room temperature. Hereafter, we will refer to the resulting samples as N-GO (GO bearing $\mathrm{N}$-aliphatic functionalities). The role of the time of treatment in the final $\mathrm{N}$ content was initially investigated by allowing the solid-gas reaction to take place during $5 \mathrm{~min}\left(\mathrm{~N}-\mathrm{GO}_{5}\right), 15 \mathrm{~min}$ $\left(\mathrm{N}-\mathrm{GO}_{15}\right), 30 \mathrm{~min}\left(\mathrm{~N}-\mathrm{GO}_{30}\right)$ and $60 \mathrm{~min}\left(\mathrm{~N}-\mathrm{GO}_{60}\right)$, while keeping the flow of ammonia constant at $300 \mathrm{~mL} \mathrm{~min}^{-1}$. Fig. 1 summarizes the $\mathrm{N}$ content of the samples, as determined by elemental analysis (blue dots; for numerical data see Table S1, ESI $\dagger$ ). Noteworthily, a substantial amount of nitrogen is already introduced with the shortest reaction time employed ( $5 \mathrm{~min}, 3.6 \mathrm{wt} \% \mathrm{~N}$ ). A slight increase in the $\mathrm{N}$ content is observed for longer times of treatment, reaching $4.4 \mathrm{wt} \% \mathrm{~N}$ after $60 \mathrm{~min}$.

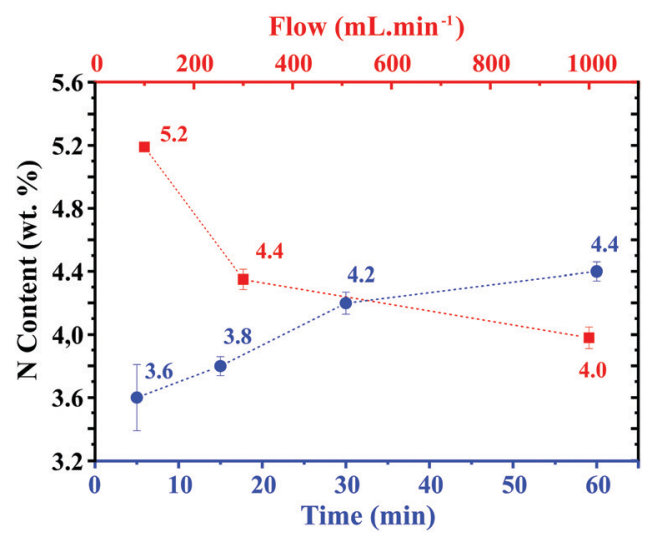

Fig. 1 Nitrogen content ( $w t \%$ ) of N-GO samples, as determined by elemental analysis. Samples have been prepared by ammonolysis treatment of GO at different times (flow rate constant at $300 \mathrm{~mL} \mathrm{~min}^{-1}$ ) and at different flow rates (time constant at $60 \mathrm{~min}$ ). 
The role of $\mathrm{NH}_{3}$ flow was also investigated and significant differences in the total $\mathrm{N}$ content were observed. Red squares in Fig. 1 correspond to the samples treated under flowing ammonia

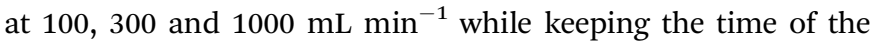
treatment constant at $60 \mathrm{~min}$. The highest $\mathrm{N}$ content is achieved at the lowest flow rate $\left(5.2 \mathrm{wt} \% \mathrm{~N}\right.$ at $\left.100 \mathrm{~mL} \min ^{-1} \mathrm{NH}_{3}\right)$. Lowering the flow rate might favor the interaction between the $\mathrm{NH}_{3}$ molecules and the GO, increasing the degree of $\mathrm{N}$-functionalisation. The formation of $\mathrm{N}$-aliphatic groups may occur via nucleophilic addition of $\mathrm{N}$ from the $\mathrm{NH}_{3}$ molecule to atoms with electrophilic character, namely carbon atoms from epoxy (ring-opening reaction) ${ }^{18}$ or carboxylic groups ${ }^{19}$ (see Scheme S1, ESI $\dagger$ ), leading respectively to the formation of amine and amide groups on the GO surface. A schematic representation of the resulting material is shown in Fig. 2a. However, as we have previously pointed out, the precise determination of the species present in graphene derivatives is not simple and complementary techniques are required for the quantification of $\mathrm{N}$-based moieties introduced within the samples after the $\mathrm{NH}_{3}$ treatment. ${ }^{6}$ The presence of amines and amides was initially assessed by X-ray diffraction (XRD) and thermogravimetric analysis (TGA) in air of the samples. XRD is a powerful tool for the analysis of $2 \mathrm{D}$ materials. ${ }^{20}$ XRD data indicate that GO does not get reduced under mild conditions of $\mathrm{NH}_{3}$ treatment employed in the present study. Fig. S1 (ESI $\dagger$ ) shows the XRD pattern of GO (black continuous line) and $\mathrm{NH}_{3}$ treated $\mathrm{GO}$ (r.t.) during $5\left(\mathrm{~N}-\mathrm{GO}_{5}\right)$ and $60 \mathrm{~min}\left(\mathrm{~N}-\mathrm{GO}_{60}\right)$. The (002) peak, characteristic of graphitic materials $\left(2 \theta=25^{\circ}\right)$ appears shifted to lower angles as it is usually observed for oxidised graphene derivatives. ${ }^{21}$ For comparison, we have

(a)

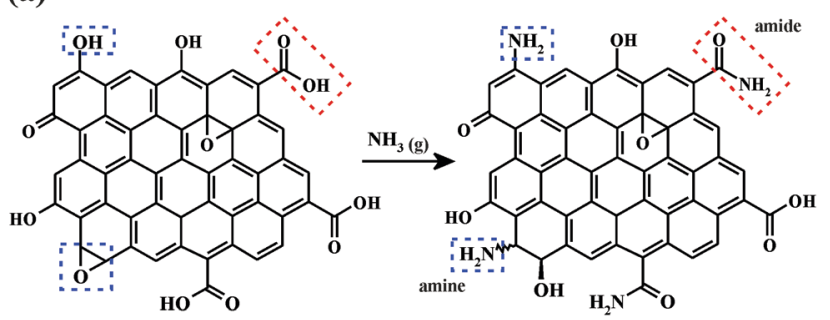

(b)

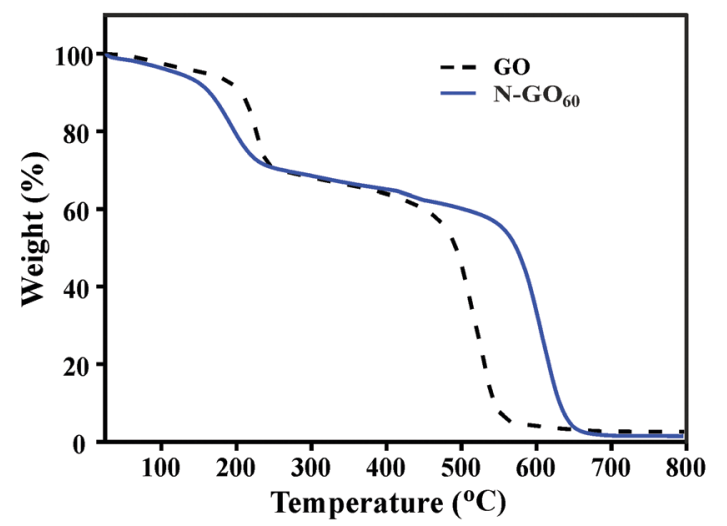

Fig. 2 (a) Schematic representation of the formation of amine and amide groups by ammonolysis treatment of $\mathrm{GO}$ at room temperature. (b) Thermogravimetric analyses in air of $\mathrm{GO}$ and $\mathrm{NH}_{3}$ treated $\mathrm{GO}$ at room temperature. TGA was performed under flowing air at a heating rate of $10{ }^{\circ} \mathrm{C} \mathrm{min}^{-1}$. additionally synthesised N-doped reduced GO (RGO) following a previously reported protocol $\left(\mathrm{NH}_{3}, 1 \mathrm{~h}, 500{ }^{\circ} \mathrm{C}\right) .{ }^{6}$ It is well established that $\mathrm{NH}_{3}$ treatment at $500{ }^{\circ} \mathrm{C}$ results in the elimination of aliphatic moieties from $\mathrm{GO}$ and induces the introduction of $\mathrm{N}$ into the lattice. As expected for RGO samples, the XRD pattern of N-doped RGO reveals the presence of the (002) peak at ca. $28^{\circ}$ (Fig. S1, ESI $\dagger$ ). Thus, XRD allows discerning in a qualitative manner between $\mathrm{N}$-aliphatic groups and $\mathrm{N}$ introduced into the lattice.

TGA also confirms this observation. As it can be seen in Fig. 2b, both GO and $\mathrm{N}-\mathrm{GO}_{60}$ show two main thermal events. An initial weight loss is observed which is attributed to the loss of aliphatic groups and a minor release of absorbed water. ${ }^{21 a}$ The lower onset of combustion temperature for $\mathrm{N}^{-\mathrm{GO}_{60}}$ is attributed to the introduction of $\mathrm{N}$-aliphatic groups that co-exist with O-aliphatic functionalities. Remarkably, a similar weight loss of ca. $30 \%$ is observed for both GO and $\mathrm{N}-\mathrm{GO}_{60}$. This is in contrast with the previous reports on $\mathrm{N}$-functionalised RGO, where a significant decrease of the aliphatic fraction is observed in the TGA data upon thermally annealing GO at $100{ }^{\circ} \mathrm{C}$ (or above) in the presence of $\mathrm{NH}_{3}$ gas. ${ }^{6,8}$ Upon ammonolysis at $100{ }^{\circ} \mathrm{C}$ or above, GO becomes reduced via the elimination of $\mathrm{CO}_{2}$ from oxidised aliphatic species (through inner rearrangements) leading to samples of reduced graphene oxide. ${ }^{6}$ The weight loss observed above $450{ }^{\circ} \mathrm{C}$ corresponds to the complete combustion of the samples which includes the most stable functionalities.

The structure of the aliphatic moieties removed during the first step of the TGA experiment can be assessed by mass spectrometry (TGA-MS). Fractions with $m / z=17,18,43,44$ and 45 are released from both GO and N-GO (Fig. 3). As it can be observed, physically absorbed water $\left(T<100{ }^{\circ} \mathrm{C}\right)$ and molecules resulting from detaching hydroxyl groups $(\mathrm{m} / \mathrm{z}=17,18)$ are present in both samples. The fragments observed above $200{ }^{\circ} \mathrm{C}$ suggest the elimination of species resulting from decomposition of O-bearing aliphatic moieties. Both homolytic cleavage and McLafferty rearrangements lead to the formation of $\mathrm{CH}_{3} \mathrm{CO}^{+}(\mathrm{m} / z=43)$, $\mathrm{CO}_{2}(\mathrm{~m} / z=44)$ and $\mathrm{CH}_{3} \mathrm{CH}_{2} \mathrm{O}^{+}(\mathrm{m} / z=45)$ fractions, typically observed in mass spectra of hydroxy, carboxylic or carbonyl containing materials. ${ }^{22}$

Although the species released by the sample treated in $\mathrm{NH}_{3}$ (N-GO) have equivalent $\mathrm{m} / \mathrm{z}$ ratios, their thermal stability is slightly lower, suggesting variations in the chemical nature of the detected fragments. The presence of N-bearing aliphatic moieties, namely amines and amides, might also result in detaching of fragments with similar $m / z$ values such as $\mathrm{NH}_{3}(m / z=17), \mathrm{NH}_{4}{ }^{+}$ $(m / z=18), \operatorname{HOCN}(m / z=43), \mathrm{OCNH}_{2}{ }^{+}(m / z=44)$ or $\mathrm{CH}_{3} \mathrm{CHNH}_{2}{ }^{+}$ $(\mathrm{m} / \mathrm{z}=45)$. The presence of wider peaks in the MS of N-GO with respect to GO suggests the release of more than one fragment with equivalent $\mathrm{m} / \mathrm{z}$. Taking into account the high amount of oxygen detected by elemental analysis in N-GO, this can be attributed to O-based aliphatic species still present in the N-GO sample after ammonolysis treatment of $\mathrm{GO}$ at room temperature.

X-ray photoelectron spectroscopy (XPS) (Fig. S2, ESI $\dagger$ ) showed a N1s peak at ca. $400 \mathrm{eV}$ in the N-GO sample. Deconvolution of this peak suggests the presence of two main functionalities, which can be attributed to amine and amide groups covalently attached to the graphitic structure (inset of Fig. S2, Table S2, ESI $\dagger$ ). 


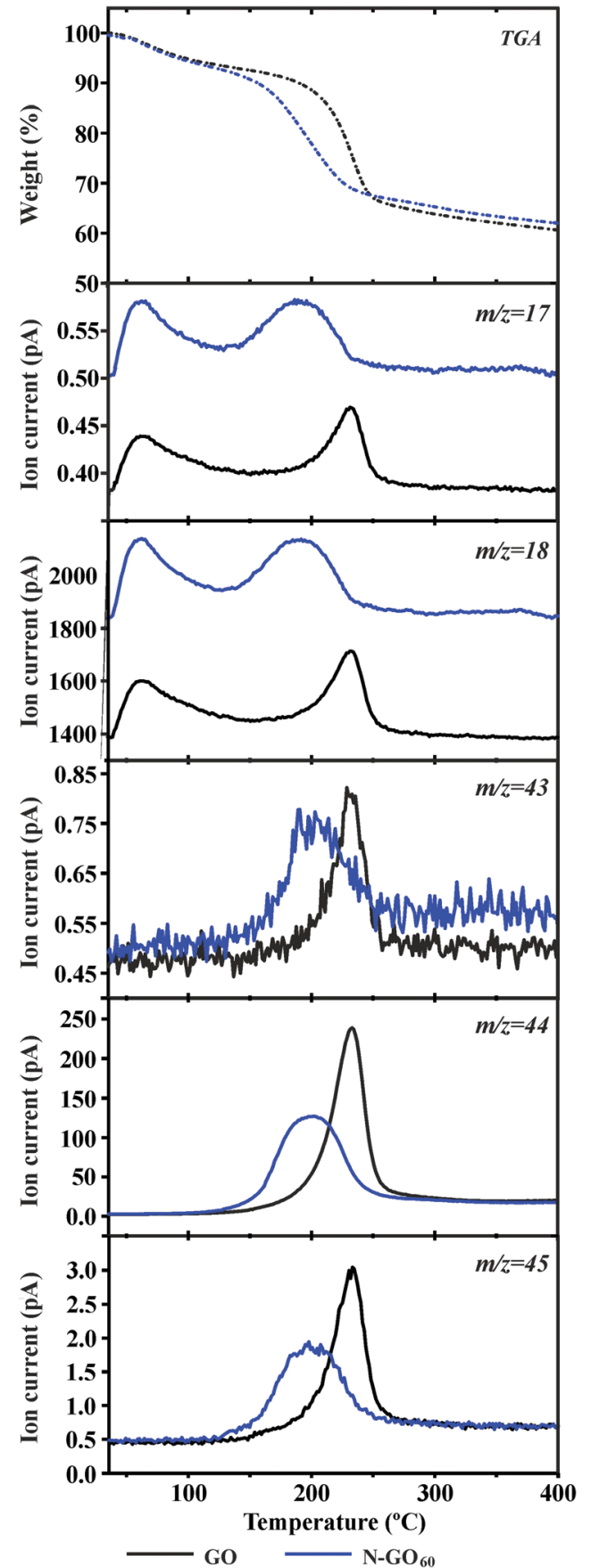

Fig. 3 TGA-MS data up to $400{ }^{\circ} \mathrm{C}$ of both $\mathrm{GO}$ and $\mathrm{N}-\mathrm{GO}(60 \mathrm{~min})$ showing the removal of $\mathrm{m} / \mathrm{z}=17, \mathrm{~m} / \mathrm{z}=18, \mathrm{~m} / \mathrm{z}=43, \mathrm{~m} / \mathrm{z}=44$ and $\mathrm{m} / \mathrm{z}=45$ fractions. Discontinuous lines (top panel) correspond to TGA data.

The presence of both amines and amides attached to GO was also confirmed by FT-IR spectroscopy (see ESI, $\dagger$ Fig. S3).

Colorimetric techniques are usually employed for the quantitative determination of $\mathrm{N}$-groups in liquid samples or soluble solids. Nevertheless, their determination on non-soluble and complex materials is problematic due to the presence of interferences, pH sensibility or low sensitivity of the technique. The Kaiser test ${ }^{23}$ was developed for checking the completeness of amino acid coupling by the qualitative determination of primary amines and has been previously used for their quantification in amino-functionalised carbon nanomaterials. ${ }^{16,24}$ The technique is based on the formation and subsequent photometric determination of Ruhemanns blue (RB), a chromophore resulting from the reaction of primary amines with ninhydrin (Scheme S2, $\mathrm{ESI} \dagger$ ). The concentration of the chromophore is proportional to the absorbance of the solution resulting from the reaction. However, the reproducibility of the results is often low, since the formation of RB is highly sensitive to the presence of interferences. In order to minimise the large deviations usually observed in the measurements, we have carried out a modified Kaiser test procedure based on a calibration curve as previously proposed by Poli et al. for the quantification of primary amino groups present in ceramic materials. ${ }^{25}$ The calibration curve was initially prepared by reaction of hexylamine with ninhydrin, leading to the formation of coloured solutions of RB with known concentrations. The resulting calibration curve (Fig. S4, ESI $\dagger$ ) has been obtained by measuring the intensity of the maximum absorbance peak of $\mathrm{RB}(c a .570 \mathrm{~nm})$ in the UV-Vis spectra. To facilitate posterior analysis, the absorbance of $\mathrm{RB}$ is directly plotted against the amine concentration because each $-\mathrm{NH}_{2}$ group leads to the formation of $1 \mathrm{RB}$ molecule upon reaction with ninhydrin. The calibration curve was initially tested using a polymeric resin standard with known amino-loading (Tentagel ${ }^{\mathrm{TM}}$-HL$\mathrm{NH}_{2}, 0.4 \mathrm{mmol} \mathrm{g}^{-1}$ ) and with an amide-containing standard (behenamide). The later was employed to discard possible interferences due to the presence of other N-based groups.

Fig. 4 shows the absorbance spectra of the solutions prepared by reaction of ninhydrin with both standards and with some selected samples. As it can be observed, samples containing $\mathrm{NH}_{2}$ groups, namely Tentagel and $\mathrm{N}-\mathrm{GO}_{60}$ show the characteristic absorbance of the RB chromophore at ca. $570 \mathrm{~nm}$, while GO and $\mathrm{N}$-doped RGO (used as control) ${ }^{6}$ do not absorb in the $500-800 \mathrm{~nm}$ range. Similarly, no absorption is detected in this spectral range for behenamide, thus confirming the selectivity of this method for the determination of amino groups. It is also worth noting that along with other complementary techniques, ${ }^{6}$ the reaction of $\mathrm{N}$-containing graphene derivatives with ninhydrin is also useful to discern between $\mathrm{N}$ introduced into the lattice and aliphatic functionalities when the latter are in the form of amines. Table S3 (ESI $\dagger$ ) shows the $\mathrm{NH}_{2}$-loading calculated from the calibration curve of the tested samples, which turns out to be $0.35 \mathrm{mmol} \mathrm{g}^{-1}$ for both $\mathrm{N}-\mathrm{GO}_{60}$ and the Tentagel ${ }^{\mathrm{TM}}-\mathrm{HL}-\mathrm{NH}_{2}$ standard. There is a good agreement, within experimental error, with the expected concentrations of $\mathrm{NH}_{2}$ groups in Tentagel ${ }^{\mathrm{TM}}-\mathrm{HL}-\mathrm{NH}_{2}$ and $\mathrm{N}-\mathrm{GO}_{60}$ (see Table S3, ESI $\dagger$ ). The amino loading in $\mathrm{N}^{-\mathrm{GO}_{60}}$ has been estimated from XPS and elemental analysis. Thus, this colorimetric test has been proven to be useful to precisely determine the amino-loading in N-containing graphene derivatives.

N-GO was finally characterised by electron microscopy. A scanning electron microscopy (SEM) image of ${\mathrm{N}-\mathrm{GO}_{60}}$ treated under ammonia at room temperature is shown in Fig. S5a (ESI $\dagger$ ). The morphology of the sheets does not seem to be modified by $\mathrm{NH}_{3}$ treatment. Transmission electron microscopy (TEM) images (Fig. S5b, ESI $\dagger$ ) and the selected area electron diffraction (SAED) pattern of the [001] plane (inset) confirm the crystalline structure 


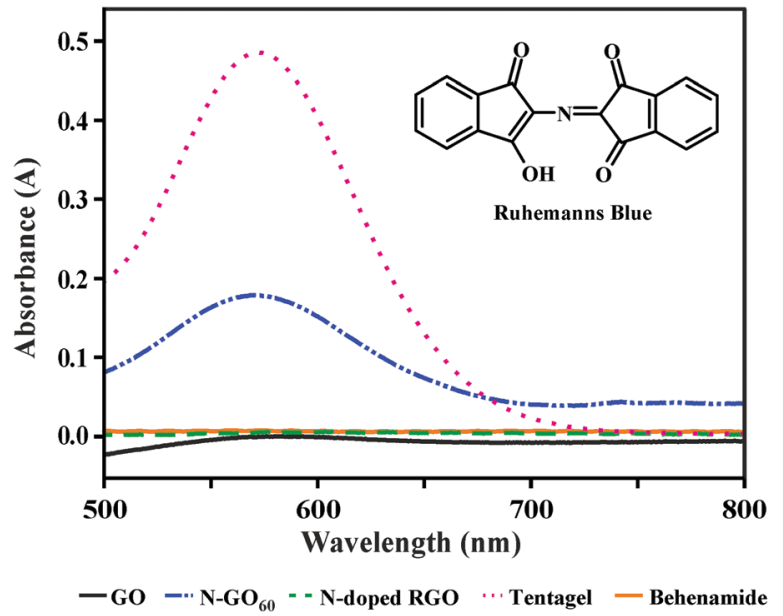

Fig. 4 UV-Vis spectra of the solutions resulting from the reaction of ninhydrin with $\mathrm{GO}, \mathrm{N}-\mathrm{GO}_{60}, \mathrm{~N}$-doped $\mathrm{RGO}$ (used as control) and the standards (Tentagel ${ }^{\mathrm{TM}}-\mathrm{HL}-\mathrm{NH}_{2}$ and Behenamide). The structure of Ruhemanns blue (RB) is included as an inset.

of this sample. The observed (100) and (110) reflections correspond to $d$-spacings of $2.12 \AA$ and $1.23 \AA$, respectively. ${ }^{26}$

To complete the study, we aimed to develop a more environmentally friendly process by decreasing the total volume of $\mathrm{NH}_{3}$ used for the synthesis. We have observed that a low $\mathrm{NH}_{3}$ flow has a positive impact on the final $\mathrm{N}$ content. Therefore, GO was placed in a hermetic system with a saturated atmosphere of $\mathrm{NH}_{3}$. Keeping the sample in a static system, in contact with $\mathrm{NH}_{3}$ during $60 \mathrm{~min}$ led to the successful $\mathrm{N}$-functionalisation of $\mathrm{GO}$ with a $\mathrm{N}$ content of $3.7 \mathrm{wt} \%$. A slight increase in the $\mathrm{N}$ content was obtained when magnetic stirring was applied to the sample (4.0 wt\%). Therefore, it is possible to functionalise GO with $\mathrm{N}$-aliphatic moieties using a remarkably reduced volume of ammonia.

To conclude, we propose a new solvent free protocol to introduce aliphatic moieties (amines and amides) onto GO at room temperature. The methodology described above allows obtaining $\mathrm{N}$-contents in the range of 3.6-5.2 wt\% with only 5 minutes of reaction or using extremely reduced amounts of ammonia gas as a nitrogen source. We have also employed a modified Kaiser test based on a calibration curve that allows a more precise quantification of the $\mathrm{NH}_{2}$-loading in $\mathrm{GO}$ derivatives than the traditional approach. The functionalisation of GO with amine and amide groups using a simple, fast and energy efficient approach greatly expands the derivatisation routes that can be employed to integrate graphene-based materials into devices and composite materials.

We acknowledge financial support through MAT2017-86616R grant and the "Severo Ochoa" Programme (SEV-2015-0496).

\section{Conflicts of interest}

There are no conflicts to declare.

\section{References}

1 (a) L. Daukiya, J. Seibel and S. De Feyter, Adv. Phys.: X, 2019, 4, 1625723; (b) J. Zhu, C. Yang, C. Lu, F. Zhang, Z. Yuan and X. Zhuang, Acc. Chem. Res., 2018, 51, 3191-3202.

2 (a) C. Martín, K. Kostarelos, M. Prato and A. Bianco, Chem. Commun., 2019, 55, 5540-5546; (b) M. Ferrándiz-Saperas, A. Ghisolfi, D. Cazorla-Amorós, C. Nájera and J. M. Sansano, Chem. Commun., 2019, 55, 7462-7465; (c) M. Rosillo-Lopez and C. G. Salzmann, $R S C$ Adv., 2018, 8, 11043-11050.

3 (a) X. Zhuang, F. Zhang, D. Wu, N. Forler, H. Liang, M. Wagner, D. Gehrig, M. R. Hansen, F. Laquai and X. Feng, Angew. Chem., Int. Ed., 2013, 52, 9668-9672; (b) X. Zhuang, Y. Mai, D. Wu, F. Zhang and X. Feng, Adv. Mater., 2015, 27, 403-427.

4 P. Wick, A. E. Louw-Gaume, M. Kucki, H. F. Krug, K. Kostarelos, B. Fadeel, K. A. Dawson, A. Salvati, E. Vázquez, L. Ballerini, M. Tretiach, F. Benfenati, E. Flahaut, L. Gauthier, M. Prato and A. Bianco, Angew. Chem., Int. Ed., 2014, 53, 7714-7718.

5 (a) D. Skidin, F. Eisenhut, M. Richter, S. Nikipar, J. Krüger, D. A. Ryndyk, R. Berger, G. Cuniberti, X. Feng and F. Moresco, Chem. Commun., 2019, 55, 4731-4734; (b) Q. Chen, R. Zhu, Q. He, S. Liu, D. Wu, H. Fu, J. Du, J. Zhu and H. He, Chem. Commun., 2019, 55, 2644-2647.

6 S. Sandoval, N. Kumar, J. Oro-Solé, A. Sundaresan, C. N. R. Rao, A. Fuertes and G. Tobias, Carbon, 2016, 96, 594-602.

7 S. Rani, M. Kumar, R. Kumar, D. Kumar, S. Sharma and G. Singh, Mater. Res. Bull., 2014, 60, 143-149.

8 S. Sandoval, N. Kumar, A. Sundaresan, C. N. R. Rao, A. Fuertes and G. Tobias, Chem. - Eur. J., 2014, 20, 11999-12003.

9 S. K. Singh, M. K. Singh, P. P. Kulkarni, V. K. Sonkar, J. J. A. Grácio and D. Dash, ACS Nano, 2012, 6, 2731-2740.

10 S. Wang, X. Li, Y. Liu, C. Zhang, X. Tan, G. Zeng, B. Song and L. Jiang, J. Hazard. Mater., 2018, 342, 177-191.

11 B. Wang, B. Luo, M. Liang, A. Wang, J. Wang, Y. Fang, Y. Chang and L. Zhi, Nanoscale, 2011, 3, 5059-5066.

12 M. S. Ahmed and Y.-B. Kim, Carbon, 2017, 111, 577-586.

13 S. Kancharla and K. Sasaki, J. Mater. Chem. A, 2019, 7, 4561-4573.

14 X. Zhuang, F. Zhang, D. Wu and X. Feng, Adv. Mater., 2014, 26, 3081-3086.

15 C.-M. Chen, Q. Zhang, X.-C. Zhao, B. Zhang, Q.-Q. Kong, M.-G. Yang, Q.-H. Yang, M.-Z. Wang, Y.-G. Yang, R. Schlögl and D. S. Su, J. Mater. Chem. A, 2012, 22, 14076-14084.

16 A. V. Isabella, R. Jésus, B. Alberto and M.-M. Cécilia, 2D Mater., 2018, 5, 035037.

17 S.-Y. Gu, C.-T. Hsieh, J.-Y. Yuan, J.-H. Hsueh and Y. A. Gandomi, Diamond Relat. Mater., 2018, 87, 99-106.

18 I. A. Vacchi, C. Spinato, J. Raya, A. Bianco and C. Ménard-Moyon, Nanoscale, 2016, 8, 13714-13721.

19 D. R. Dreyer, S. Park, C. W. Bielawski and R. S. Ruoff, Chem. Soc. Rev., 2010, 39, 228-240.

20 M. Dopita, M. Rudolph, A. Salomon, M. Emmel, C. G. Aneziris and D. Rafaja, Adv. Eng. Mater., 2013, 15, 1280-1291.

21 (a) A. Alazmi, S. Rasul, S. P. Patole and P. M. F. J. Costa, Polyhedron, 2016, 116, 153-161; (b) J. Smajic, A. Alazmi, N. Batra, T. Palanisamy, D. H. Anjum and P. M. F. J. Costa, Small, 2018, 14, 1803584.

22 F. W. McLafferty, Mass Spectrometry of Organic ions, A. P. Inc., 1963.

23 E. Kaiser, R. L. Colescott, C. D. Bossinger and P. I. Cook, Anal. Biochem., 1970, 34, 595-598.

24 (a) C. Samorì, R. Sainz, C. Ménard-Moyon, F. M. Toma, E. Venturelli, P. Singh, M. Ballestri, M. Prato and A. Bianco, Carbon, 2010, 48, 2447-2454; (b) M. Quintana, K. Spyrou, M. Grzelczak, W. R. Browne, P. Rudolf and M. Prato, ACS Nano, 2010, 4, 3527-3533.

25 E. Poli, V. Chaleix, C. Damia, Z. Hjezi, E. Champion and V. Sol, Anal. Methods, 2014, 6, 9622-9627.

26 J. C. Meyer, A. K. Geim, M. I. Katsnelson, K. S. Novoselov, D. Obergfell, S. Roth, C. Girit and A. Zettl, Solid State Commun., 2007, 143, 101-109. 